\section{Epigenetische Therapie vs. Chemotherapie bei Patienten mit AML ab 65 Jahren}

\begin{abstract}
Die Prognose älterer AML-Patienten bei konventioneller Induktionstherapie ist schlecht. Autoren verglichen Überlebensraten unter Chemotherapie und unter Substanzen, die eine DNA-Strang-Demethylierung bewirken.
\end{abstract}

Ein ne Studiengruppe des MD Anderson Cancer Center in Texas analysierte Daten von insgesamt 671 Patienten im Alter von mindestens 65 Jahren, die an ihrer Klinik wegen neu aufgetretener akuter myeloischer Leukämie (AML) behandelt worden waren.

In Gruppe $1(\mathrm{n}=557)$ hatten die Patienten zur Induktion eine auf hochdosiertem Cytarabin basierende Chemotherapie erhalten, meist in Verbindung mit Idarubicin. Die Patienten der Gruppe 2 ( $n=114$ ) waren mit epigenetisch wirksamen Substanzen behandelt worden: In 67 Fällen wurde Decitabin, in 47 Azacitidin verabreicht - allein oder in Kombination mit Histon-Deacetylase-Inhibitoren.
Zwischen beiden Gruppen bestanden geringe, wenn auch signifikante Unterschiede bezüglich des Alters (71 vs. 74 Jahre) und bei den Parametern Hämoglobin ( 8,3 vs. $8,7 \mathrm{~g} / \mathrm{dl})$, Blasten im Knochenmark (43 vs. $36 \%$ ) und Blasten im peripheren Blut (15 vs. $7 \%$ ). Bezüglich ungünstiger zytogenetischer Befunde und des ECOG-Performance-Status unterschieden sie sich jedoch nicht signifikant.

Bei den Raten für Komplettremissionen ergab sich noch ein signifikanter Vorteil zugunsten der Chemotherapie mit 42 vs. $28 \%(\mathrm{p}=0,001)$. Die Mortalitätsraten innerhalb der ersten acht Wochen wiesen jedoch bei $18 \%$ gegen- über $11 \%(p=0,075)$ auf einen positiven Trend für die epigenetische Therapie hin. Das rezidivfreie Überleben (12,0 vs. 10,5 Monate) und das mediane Gesamtüberleben (6,7 vs. 6,5 Monate) unterschieden sich nicht signifikant.

In der multivariaten Analyse waren Parameter wie Alter, ungünstige Zytogenetik oder Blastenanteil unabhängige Risikofaktoren, nicht jedoch die Art der Therapie.

Fazit: Die Studienteilnehmer zeigten unter konventioneller Chemotherapie zwar häufiger Komplettremissionen, dafür aber im Trend eine höhere Mortalitätsrate bis zur achten Woche. Die epigenetische Therapie bot vergleichbare Ergebnisse im Langzeitverlauf.

Andreas Fischer

Quintás-Cardama A et al. Epigenetic therapy is associated with similar survival compared with intensive chemotherapy in older patients with newly diagnosed acute myeloid leukemia. Blood. 2012;120(24):4840-5.

\title{
Therapieoptionen bei AML älterer Patienten erweitert? Bortezomib vielversprechend
}

\section{Seit Jahrzehnten ist bei der Induktionstherapie der AML die Kombination Cytarabin plus Anthrazyklin Standard. Nun wurde bei älteren Patienten der zusätzlichen Einsatz von Bortezomib - in der Induktionstherapie wie auch dosisabhängig bei der Konsolidierung - untersucht.}

A n der Studie nahmen 95 Patienten im Alter von 60-75 Jahren teil, die an einer nicht vorbehandelten akuten myeloischen Leukämie (AML) erkrankt waren. Auch Patienten mit therapieinduzierter AML und myelodysplastischem Syndrom wurden in die Studie eingeschlossen. Das mediane Alter lag bei 67 Jahren.

Zur Induktionstherapie erhielten die Patienten Cytarabin in einer Dosierung von $100 \mathrm{mg} / \mathrm{m}^{2}$ während der ersten sieben Zyklustage sowie Daunorubicin (60 $\mathrm{mg} / \mathrm{m}^{2}$ ) an den ersten drei Tagen. Vor Gabe der Chemotherapeutika wurde Bortezomib mit $1,3 \mathrm{mg} / \mathrm{m}^{2}$ an den Tagen 1, 4, 8 und 11 als Bolus verabreicht.

Bei Studienteilnehmern mit kompletter Remission erfolgte eine Konsolidierungstherapie mit bis zu zwei Zyklen,

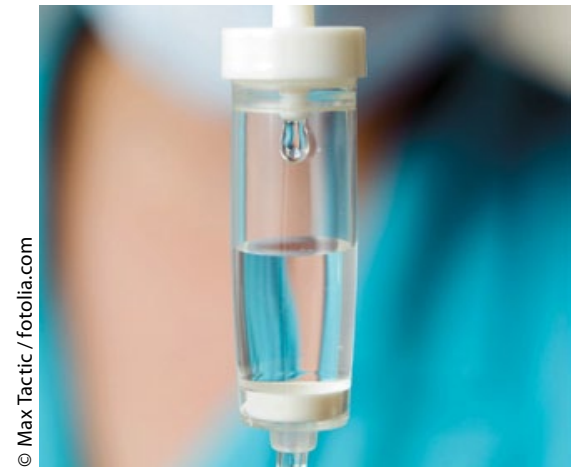

bestehend aus $2 \mathrm{mg} / \mathrm{m}^{2}$ Cytarabin an Tag 1-5 zusammen mit Bortezomib in ansteigenden Dosierungen von 0,7, 1,0 und $1,3 \mathrm{mg} / \mathrm{m}^{2}$.

62 der 95 Studienteilnehmer (65\%) erreichten unter der Induktionstherapie eine komplette Remission. Bei vier wei- teren Patienten ergab sich eine komplette Remission mit einer nicht ausreichenden Erholung der Thrombozytenzahlen $(<100.000 / \mu l)$.

Während der Konsolidierung trat bei zwei Patienten unter der BortezomibDosierung von $1,3 \mathrm{mg} / \mathrm{m}^{2}$ eine Toxizität auf, die gemäß Protokoll eine Dosisreduktion bzw. Medikationsabbruch erforderlich machte. Insgesamt elf Studienteilnehmer entwickelten eine sensorische Neuropathie vom Grad 3 - davon neun während der Induktionsphase.

Das mediane krankheitsfreie Überleben lag bei acht Monaten, das mediane Gesamtüberleben betrug zwölf Monate.

Fazit: Die Remissionsraten unter der Induktion mit zusätzlicher Gabe von Bortezomib sehen die Autoren als ermutigend an. Sensorische Neuropathien mit einer Grad-3-Toxizität traten bei elf $\mathrm{Pa}$ tienten auf.

Andreas Fischer

Attar EC et al. Bortezomib added to daunorubicin and cytarabin during induction therapy and to intermediate-dose cytarabine for consolidation in patients with previously untreated acute myeloide leukemia 60 to 75 years: CALGB (Alliance) Study 10502. J Clin Oncol.

2013;31(7):923-9. 